\title{
Freezing a Coherent Field Growth in a Cavity by Quantum Zeno Effect
}

\author{
J. Bernu ${ }^{1}$, S. Deléglise ${ }^{1}$, C. Sayrin ${ }^{1}$, S. Kuhr ${ }^{1}$ I. Dotsenko ${ }^{1,2}$, M. Brune ${ }^{1}$ J. M. Raimond ${ }^{1}$, and S. Haroche $e^{1,2}$ \\ ${ }^{1}$ Laboratoire Kastler Brossel, Ecole Normale Supérieure, CNRS, \\ Université P. et M. Curie, 24 rue Lhomond, F-75231 Paris Cedex 05, France \\ ${ }^{2}$ Collège de France, 11 Place Marcelin Berthelot, F-75231 Paris Cedex 05, France
}

(Dated: November 2, 2018)

\begin{abstract}
We have frozen the coherent evolution of a field in a cavity by repeated measurements of its photon number. We use circular Rydberg atoms dispersively coupled to the cavity mode for an absorption-free photon counting. These measurements inhibit the growth of a field injected in the cavity by a classical source. This manifestation of the Quantum Zeno effect illustrates the back action of the photon number determination onto the field phase. The residual growth of the field can be seen as a random walk of its amplitude in the two-dimensional phase space. This experiment sheds light onto the measurement process and opens perspectives for active quantum feedback.
\end{abstract}

PACS numbers: $03.65 . \mathrm{Ta}, 03.65 . \mathrm{Xp}$, 42.50.Pq

The 'Quantum Zeno' effect is a spectacular manifestation of the measurement back action. The coherent evolution of a quantum system is inhibited by repeated projective measurements performed at short time intervals. This effect, theoretically discussed in [1, 2, 3], has been observed on the evolution of two-level systems such as molecules [4], trapped ions [5], cold atoms [6] as well as on polarization of light [7]. We describe here the observation of the Zeno effect on a harmonic oscillator. The build-up of a coherent field in a high- $Q$ superconducting cavity coupled to a classical source is inhibited by watching repeatedly the photon number. The measurements use circular Rydberg atoms as non-destructive probes [8, 9]. The system free evolution is here a run-away coherent classical process, instead of the two-level quantum dynamics considered in [4, 5, 6, 7]. When watching the system, we record single trajectories and the field state evolution is obtained by a statistical analyzis of these data. This study sheds light on the relationship between the Zeno effect and the measurement back action.

We first revisit the basics of the Zeno effect in the context of our experiment. A classical source is resonantly coupled to the cavity. The evolution due to the source-cavity coupling during a time $T$ is described by the displacement operator $D(\alpha)=\exp \left(\alpha a^{\dagger}-\alpha^{*} a\right)$ [10], where $\alpha=\lambda T, a\left(a^{\dagger}\right)$ are the photon annihilation (creation) operators and $\lambda$ is the complex amplitude of the source. At this stage, we neglect relaxation, assuming $T \ll T_{c}$, where $T_{c}$ is the cavity damping time. The initially empty cavity contains at time $T$ the coherent state

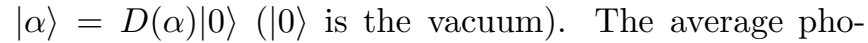
ton number, $\bar{n}=|\alpha|^{2}=|\lambda|^{2} T^{2}$, runs away quadratically with $T$. This evolution can be split into small steps of duration $\Delta t \ll T$. The final displacement results from the coherent accumulation of successive injection pulses,

*Present address: Johannes Gutenberg Universität, Institut für Physik, Staudingerweg 7, D-55128 Mainz, Germany

${ }^{\dagger}$ Electronic address: brune@lkb.ens.fr each being described by the translation operator $D(\lambda \Delta t)$.

We now watch the field evolution. In order to separate the field probing from its coupling with the source, we alternate measurements with injection pulses of duration $\Delta t$, such that $|\lambda| \Delta t \ll 1$. After the first pulse, the cavity contains a coherent state $|\lambda \Delta t\rangle \approx|0\rangle+$ $\lambda \Delta t|1\rangle(|1\rangle$ is the one-photon state). With a probability $p_{0} \approx 1-(|\lambda| \Delta t)^{2}$, the first measurement projects this state back onto $|0\rangle$. After $N$ iterations of the injection/measurement sequence, the field is left in $|0\rangle$ with a probability $p_{0}^{N} \approx\left[1-(|\lambda| \Delta t)^{2}\right]^{N} \approx 1-|\lambda|^{2} T \Delta t$, where $T=N \Delta t$ is the total injection time (we assume that $\Delta t$ is chosen small enough so that $\left.|\lambda|^{2} T \Delta t \ll 1\right)$. The average photon number, $\bar{n}=|\lambda|^{2} T \Delta t$, grows linearly with $T$. This is strikingly different from the quadratic growth obtained without measurements. The final mean photon number is smaller than $|\lambda|^{2} T^{2}$, the value reached without measurements. The corresponding reduction factor, $T / \Delta t$, can be made arbitrarily large. At the limit of infinitely many measurements $(\Delta t \rightarrow 0$ and $N \rightarrow \infty$ with $T$ constant), the field remains in $|0\rangle[11]$.

An interesting insight into the Zeno effect on light is provided by considering the back action of the measurement on the field phase. Each photon number determination erases phase information, randomizing at each step the field phase. The field amplitude undergoes a two-dimensional random walk, with a step size $|\lambda| \Delta t$, instead of a deterministic addition of identical displacements along a fixed direction. The final r.m.s. amplitude, $\sqrt{N}|\lambda| \Delta t$, corresponding to $\bar{n}=|\lambda|^{2} T \Delta t$, coincides with the prediction based on the projection postulate.

In the analyzis of the Zeno effect, it is essential that the probability for finding the system in its initial state has a quadratic evolution at short times. There is no Zeno effect when the cavity is coupled to an environment with a short memory time $\tau_{c}$, inducing an evolution with probabilities varying linearly with time. Assume for instance that the cavity, prepared in vacuum, relaxes within the damping time $T_{c}$ towards a thermal equilibrium with a blackbody mean photon number $n_{b}$ [9]. The probability for finding the cavity in $|0\rangle$ after 


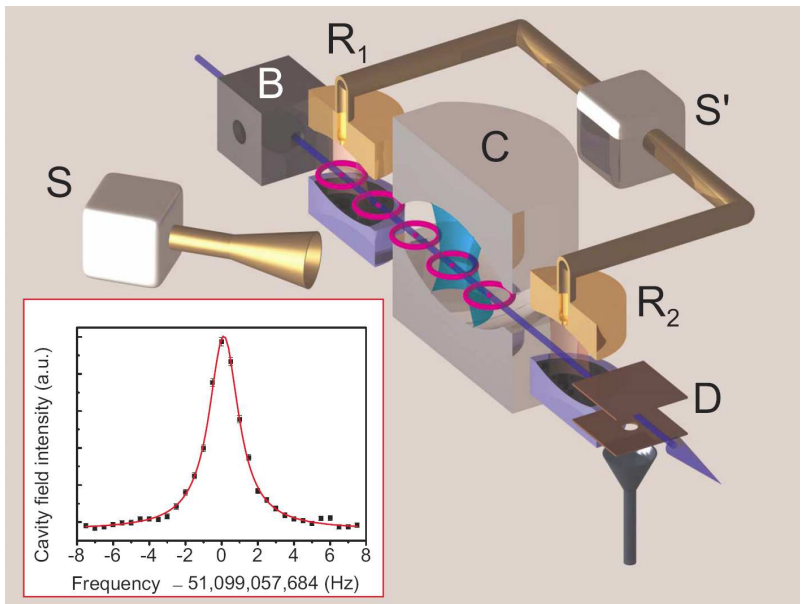

FIG. 1: Experimental set-up (cavities $C, R_{1}$ and $R_{2}$ are cut for clarity). The inset shows the cavity spectrum (dots) and a lorentzian fit (line) with a $2.0 \mathrm{~Hz}$ FWHM.

an initial time interval $\Delta t \gg \tau_{c}$ is $p_{0} \approx 1-\left(n_{b} / T_{c}\right) \Delta t$, provided $n_{b} \Delta t / T_{c} \ll 1$. The probability for being in $|0\rangle$ after $N$ steps of length $\Delta t$, each followed by a measurement, is then $p_{0}^{N} \approx\left[1-\left(n_{b} / T_{c}\right) \Delta t\right]^{N} \approx 1-n_{b} T / T_{c}$ (with $\left.n_{b} T / T_{c} \ll 1\right)$. The field energy grows linearly at short times, with the same rate as without measurements.

Similarly, the exponential damping of a field stored in the cavity leads to state probabilities varying linearly with time, which are not modified by repeated measurements. The Zeno effect thus does not affect the irreversible damping rate $T_{c}$ of the cavity. Note that other irreversible systems may exhibit at short, but experimentally accessible times, a non-exponential behaviour with a quadratic initial evolution. Quantum Zeno and antiZeno effects have been theoretically described [12] and observed [13] for such irreversible processes.

Our experimental set-up [10, 14] is shown in figure 1. The key element is the superconducting cavity $C$, made up of two niobium mirrors facing each other [15]. It has a remarkably long damping time $T_{c}=0.13 \mathrm{~s}$, when cooled to $0.8 \mathrm{~K}$. The pulsed classical source $S$, tuned to resonance with the cavity mode at $51.099 \mathrm{GHz}$, irradiates $C$ from the side. A small fraction of the photons emitted in a pulse is coupled into $C$ through diffraction on the mirrors edges. The other photons disappear quasiinstantaneously when $S$ is switched off.

Phase stability is critical for the coherent accumulation of successive pulses in $C$. An atomic clock stabilizes $S$ to a sub-Hertz frequency width and stability. The lifetime-limited linewidth of $C$ is $1.2 \mathrm{~Hz}$ full width at half maximum (FWHM). The cavity frequency must be stabilized to this level, requiring a control of the $2.7 \mathrm{~cm}$ cavity length to a 0.5 picometer precision. We stabilize the cavity length passively. The cryostat has been isolated from external vibrations. We have stabilized the refrigerator temperature to $\pm 10^{-4} \mathrm{~K}$ and the pressure of the liquid helium bath to \pm 0.1 mbar. Piezo elements tune the mirrors separation and hence the mode frequency with a
$2.4 \mathrm{kHz} / \mathrm{V}$ sensitivity. Their drive voltage $(83 \mathrm{~V})$ is stabilized to $\pm 0.1 \mathrm{mV}$ and drifts by less than $\pm 0.2 \mathrm{mV} / \mathrm{h}$.

The inset in figure 1 shows the cavity resonance spectrum probed by $S$ operating in a quasi-continuous emission mode. We switch off $S$ for short time intervals, during which the field intensity in $C$ is measured by resonant Rydberg atoms [15]. These atoms are not submitted to the intense field radiated outside $C$ when $S$ is on. The Lorentzian fit FWHM is $2.0 \mathrm{~Hz}$, differing from the expected $1.2 \mathrm{~Hz}$ width, due to a combination of short term fluctuations and long term drift. During data acquisition, we measure the cavity frequency every ten minutes and reset $S$ at resonance with $C$. If the drift between two measurements is above $1.2 \mathrm{~Hz}$, we eliminate the corresponding data. We estimate that the $S-C$ detuning $\delta / 2 \pi$ is, on average, $0.6 \pm 0.2 \mathrm{~Hz}$ during data acquisition.

The non-resonant QND probe atoms are prepared in box $B$ from a velocity-selected rubidium atomic beam (velocity $v=250 \pm 1 \mathrm{~m} / \mathrm{s}$ ) in the circular Rydberg level $g$ (principal quantum number 50). Rydberg atom samples are prepared within $2 \mu$ s pulses. Their position is wellknown during their flight through the apparatus. The cavity mode is close to resonance with the transition from $g$ to $e$ (circular state with principal quantum number 51). The atom-cavity detuning, $\Delta / 2 \pi=240 \mathrm{kHz}$, is larger than the vacuum Rabi frequency, $\Omega_{0} / 2 \pi=50 \mathrm{kHz}$, which defines the atom-cavity coupling. The atoms are transparent probes, unable to absorb or emit photons in $C$. The $g \rightarrow e$ transition frequency is, however, lightshifted. This shift, proportional to the photon number $n$, is used to measure it non-destructively $[8,9]$.

Before entering $C$, each atom is prepared in the superposition $(|e\rangle+|g\rangle) / \sqrt{2}$ by a $\pi / 2$-pulse, resonant on the $g \rightarrow e$ transition, produced by the classical source $S^{\prime}$ in the low- $Q$ cavity $R_{1}$. Using the Bloch pseudo-spin representation, we describe this superposition as a spin in the $O X$ direction of the Bloch sphere. The light shift accumulated during the atom-cavity interaction changes the phase of the $e / g$ superposition, in a frame rotating at the atomic transition frequency in vacuum [9, 10]. When $n$ photons are stored in $C$, the atomic spin rotates by an angle $n \Phi_{0}$ in the equatorial plane of the sphere. With the chosen values of $\Delta$ and $v$, the phase shift per photon is $\Phi_{0} \approx \pi / 4$. We measure the spin direction by applying on the atom, at the exit of $C$, a $\pi / 2$-pulse in $R_{2}$ with a phase $\varphi$ relative to that of the $R_{1}$ pulse. The subsequent detection of the atomic state ( $e$ or $g$ ) in the field-ionization detector $D$ is equivalent to a spin detection at the exit of $C$ along the $O u$ axis in the equatorial plane, at an angle $\varphi$ with $O X$.

The setting $\Phi_{0} \approx \pi / 4$ is appropriate for a QND measurement up to $n=7$ [9]. The photon numbers $n=0 \ldots 7$ are associated to 8 directions of the atomic spin at the exit of $C$. We send an ensemble of probe atoms in a time shorter than $T_{c}$. For a given photon number $n$, all atomic spins in this ensemble point in the same direction. We infer it by a quantum tomography process, in which we detect the spins individu- 


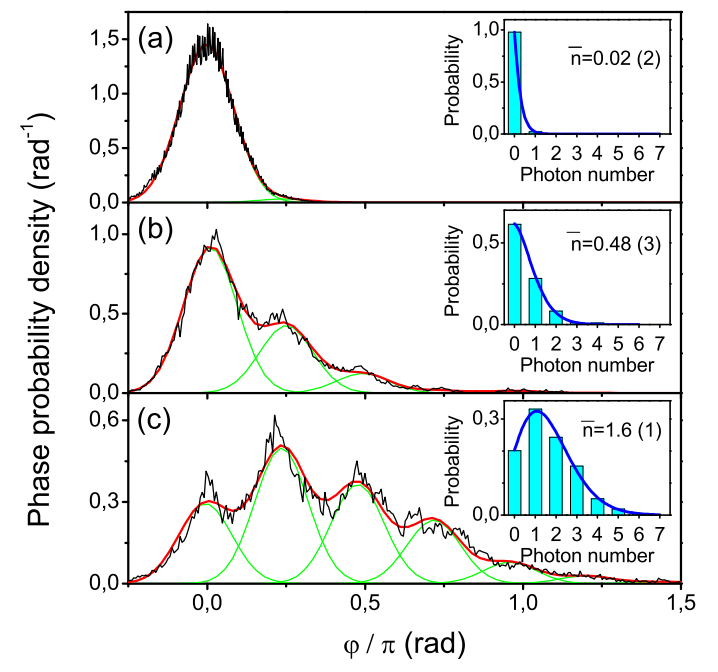

FIG. 2: Probability distributions of the atomic spin phase after 0 (a), 20 (b) and 50 (c) field injections in $C$ (no intermediate QND measurements). The thick red line is a fit to a sum of gaussian distributions (thin green lines). Resolved peaks are centered at the quantized light shifts, clearly illustrating field energy quantization. The gaussian fits provide a direct measurement of the photon number probability distributions shown in the insets, together with poissonian fits (solid lines).

ally along one out of four different axes corresponding to $\varphi / \pi=-0.250,-0.047,+0.247$ and +0.547 . With 110 atoms in the ensemble, the statistical noise on the tomography process is of the order of the phase shift $\Phi_{0}$.

Let us first examine the coherent field growth without QND probe atoms between the injections. An experimental sequence starts by reseting the field to $|0\rangle$ using a large number of absorbing atoms, prepared in $g$ and set at resonance with $C$ via the Stark effect. We then inject $N$ identical pulses (50 $\mu$ s duration) in $C$, separated by a time interval $T_{i}=5.04 \mathrm{~ms}$. At the end of the sequence, we perform a QND measurement of the cavity field.

Figure 2(a-c) presents the probability distributions of the measured spin phase $\varphi$ for $N=0,20$ and 50 injection pulses. For each sequence, we detect $\approx 200$ atoms over a $T_{m}=72 \mathrm{~ms}$ duration, starting $2 T_{i}$ after the last injection. From these data, we extract about 90 non-independent $\varphi$ values based on spin tomographies performed on a sliding window containing an ensemble of 110 atomic detections. We repeat the procedure 500 to 2000 times. For $N=0$, the phase distribution is peaked at $\varphi=0$ revealing an empty cavity. For $N=20$, the peaks centered at $\Phi_{0}$ and $2 \Phi_{0}$ reveal the build-up of small probabilities for having one and two photons. For $N=50$ injections, equidistant peaks are visible, corresponding to $n=0 \ldots 5$. These discrete peaks directly reveal the field energy quantization in $C$ [16]. The phase distributions are fitted by sums (thick red line) of Gaussians (thin green lines) with a

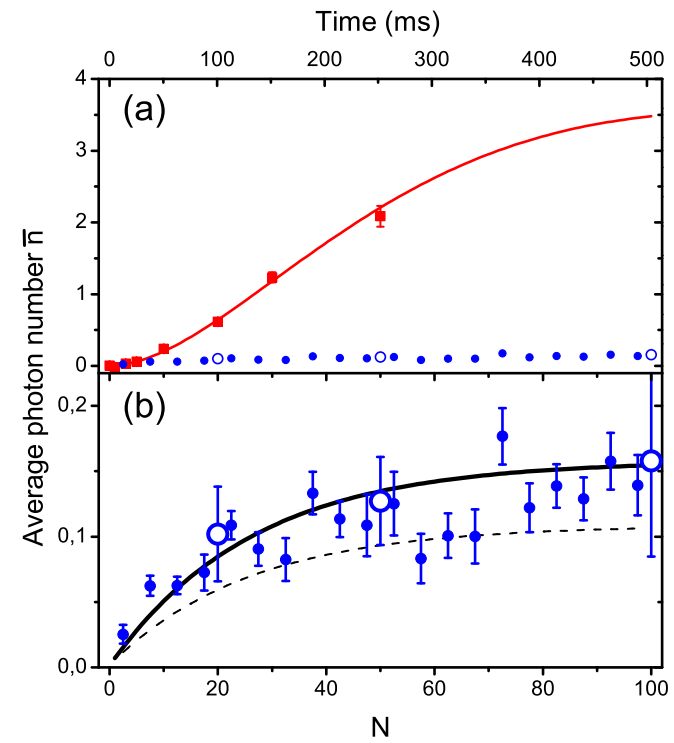

FIG. 3: (a) Average photon number $\bar{n}$ in $C$ as a function of the number $N$ of injection pulses (bottom axis). The top axis gives a time scale for the complete sequence, including injection and measurement times. The red squares correspond to the uninhibited field growth, without measurements between injections (statistical error bars for $N<50$ are smaller than point size). The initial evolution is quadratic. The line is a fit providing a precise calibration of the injection pulse amplitude. The blue dots result from atomic spin tomography performed between injection pulses. The field growth is inhibited by the quantum Zeno effect. The open circles correspond to QND measurements of the photon number using 200 atomic detections at the end of sequences with 20,50 and 100 injection/measurement cycles. (b) Inhibited field evolution with an expanded vertical scale (blue dots in a). The error bars reflect the statistical noise. The solid and dotted black lines are theoretical predictions (see text). The initial growth is now linear.

unique standard deviation of $0.266 \pi$, corresponding to the spin tomography statistical noise. From these fits, we extract $\Phi_{0}=0.233 \pi$. We also obtain the photon number distributions and their averages. These distributions are poissonian as expected for coherent states, see insets in figure 2 ,

Cavity relaxation cannot be totally neglected during the delay between the last injection and the measurement. We thus correct the measured mean photon number by a scaling factor 1.4, taking into account the $\approx 2 T_{i}+T_{m} / 2$ delay between the last injection pulse and the center of the measurement time interval. Figure 3 (a) shows the evolution of the rescaled average photon number $\bar{n}$ as a function of $N$ (red squares). Phase stability problems prevented us from recording data for $N>50$. As expected, the initial growth is quadratic. For large $N$ values, the sequence duration is comparable to $T_{c}$ and 
$1 / \delta$. For a comparison with theory, we must take into account cavity damping and cavity-source detuning. The expected cavity field amplitude after $k$ injections is:

$\alpha_{k}=e^{-T_{i} / 2 T_{c}} \alpha_{k-1}+e^{i(k-1) T_{i} \delta} \alpha_{1}=\frac{e^{-k T_{i} / 2 T_{c}}-e^{i k \delta T_{i}}}{e^{-T_{i} / 2 T_{c}}-e^{i \delta T_{i}}} \alpha_{1}$,

where $\alpha_{1}$ is the first pulse amplitude, which is taken as the phase reference ( $\alpha_{1}$ assumed to be real, without loss of generality). The value of $\alpha_{1}$ is deduced from a fit of the calculated photon number with the data (solid line in figure $3(\mathrm{a})$ ), taking into account the estimated $\delta$ value. We get $\bar{n}_{1}=\alpha_{1}^{2}=0.00223 \pm 0.00012$.

We now send QND probe atoms between injections and record their final state thus acquiring information from each realization of the field state. In order to determine the evolution of the average photon number, we perform a statistical analyzis of many such realizations, using a simplified version of the spin tomography method described above. Since the photon number is now expected to remain small, we use a single detection phase, $\varphi=-0.278 \pi$, which optimally discriminates the spin directions corresponding to 0,1 and 2 photons. To obtain the spin histogram and thus determine the average photon number at a given time, we collect the information from atoms detected in a $25 \mathrm{~ms}$ window around this time ( 50 atoms detected during 5 injection/measurement cycles) and repeat the procedure from 500 to 2500 times. The basic conclusions of our initial qualitative discussion are valid, since the amplitude injected by five consecutive pulses is still very small.

The corresponding average photon number, $\bar{n}$, is shown (blue dots) as a function of $N$ in figure [3(a). All $\bar{n}$ values are determined from independent samples made of 50 detected atoms. We check this simplified measurement by a complete spin tomography performed on $\approx 200$ detected atoms at the end of sequences with 20, 50 and 100 injection/measurement cycles (open circles in figure 3(a)). The field growth is almost completely inhibited, $\bar{n}$ remaining below 0.2 .

Figure 3(b) presents the inhibited field growth with an expanded vertical scale. This growth can be modelled by a random walk, assuming that the field phase is blurred between two injections. The dotted black line in figure 3) (b) presents the prediction of this model with no adjustable parameter. This prediction is slightly below the observations, indicating that phase is not totally blurred between injections. We have performed a detailed quantum Monte Carlo simulation of the field phase diffusion induced by the atoms crossing $C$. It includes the cavity relaxation, the finite $0.8 \mathrm{~K}$ temperature of its mode $\left(n_{b}=0.05\right.$ blackbody photons on average) and the finite detection efficiency of $D(50 \%)$. The simulation results (solid black line in figure 3(b)) are in excellent agreement with the observations and clearly exhibit the initial linear growth of $\bar{n}$, characteristic of the Zeno effect.

We have observed a clear manifestation of the quantum Zeno effect on light. Repeated intensity measurements performed on the field in a high- $Q$ cavity inhibit the quadratic run-away of the energy fed by a pulsed classical source. The residual field growth is modelled by a random walk in phase space. This effect illustrates vividly the back action of the photon number measurement onto the field phase. The realization of this experiment opens interesting perspectives for controlling quantum systems. Instead of freezing their evolution, repeated measurements could provide information used to channel them towards tailored quantum states by active feed-back operations [17].

Acknowledgements This work was supported by the Agence Nationale pour la Recherche (ANR), by the Japan Science and Technology Agency (JST), and by the EU under the IP project SCALA. S.D. is funded by the Délégation Générale à l'Armement (DGA).
[1] B. Misra and E. C. G. Sudarshan, J. Math. Phys. Sci. 18, 756 (1977); K. Koshino and A. Shimizu, Phys. Rep. 412, 191 (2005) and references therein.

[2] D. Home and A. A. B. Whitaker, Annals of Physics 258, 237 (1997).

[3] H. Nakazato M. Namiki, S. Pascazio and H. Rauch, Phys. lett. A 217, 203 (1996).

[4] B. Nagels, L. J. F. Hermans and P. L. Chapovsky, Phys. Rev. Lett. 79, 3097 (1997).

[5] W. M. Itano, D. J. Heinzen, J. J. Bollinger and D. J. Wineland, Phys. Rev. A 41, 2295 (1990); Chr. Balzer, R. Huesman, W. Neuhauser and P. Toschek, Opt. Comm. 180, 115 (2000).

[6] E. W. Streed et al., Phys. Rev. Lett. 97, 260402 (2006).

[7] P. G. Kwiat et al., Phys. Rev. Lett. 83, 4725 (1999); O. Hosten et al., Nature 439, 949 (2006).

[8] S. Gleyzes et al., Nature 446, 297 (2007).

[9] C. Guerlin et al., Nature 448, 889 (2007).

[10] S. Haroche and J. M. Raimond, Exploring the Quantum
(Oxford University Press, Oxford, 2006).

[11] This analysis of the Zeno effect overlooks events in which the field ends up in vacuum after beeing transiently excited. For $N \rightarrow \infty$ the probability of such events, which involves terms of order $|\lambda|^{4} \Delta t^{4}$, is negligibly small. See [3] for an analyzis of a similar problem in the Zeno effect of a two-level system.

[12] A. G. Kofman and G. Kurizki, Nature 405, 546 (2000); P. Facchi, H. Nakazato, and S. Pascazio, Phys. Rev. Lett. 86, 2699 (2001).

[13] M. C. Fischer, B. Gutiérrez-Medina and M. G. Raizen, Phys. Rev. Lett. 87, 040402 (2001).

[14] J. M. Raimond, M. Brune, and S. Haroche, Rev. Mod. Phys. 73, 565 (2001).

[15] S. Kuhr et al., Appl. Phys. Lett. 90, 164101 (2007).

[16] D. I. Schuster et al., Nature 445, 515 (2007).

[17] J. M. Geremia, Phys. Rev. Lett. 97, 073601 (2006). 